\title{
Minor Influence of Myopic Laser In Situ Keratomileusis on the Posterior Corneal Surface
}

\author{
Alfonso Pérez-Escudero, ${ }^{1}$ Carlos Dorronsoro, ${ }^{1}$ Lucie Sawides, ${ }^{1}$ Laura Remón, ${ }^{1}$ \\ Jesús Merayo-Lloves, ${ }^{2}$ and Susana Marcos ${ }^{1}$
}

Purpose. To check whether myopic LASIK induces changes on the posterior corneal surface.

Methods. A Scheimpflug system (Pentacam; Oculus, GmbH, Wetzlar, Germany) was used to measure preoperative and postoperative posterior corneal topography in 27 eyes (of 14 subjects) that had undergone standard myopic LASIK surgery (attempted corrections between -1.25 and $-8.50 \mathrm{D}$ ) and on 18 nonoperated eyes ( 9 subjects). A hybrid porcine-plastic eye model was developed to validate the measurement technique. Longitudinal displacement of the posterior corneal apex and changes of the apical radius of curvature and asphericity were computed.

Results. Measurements on a hybrid model eye of known posterior corneal geometry showed that the measured posterior corneal radius of curvature was minimally affected by the geometry of the anterior surface. The measurements on patients showed that, on average, the only relevant (though clinically unimportant) change in radius of curvature and asphericity occurred the first day after surgery $(\Delta R=-28 \pm 34$ $\mu \mathrm{m}$ and $\Delta Q=-0.06 \pm 0.06$ ). No statistically significant change was observed afterward. The change in radius was more pronounced in the vertical direction than in the horizontal direction. On average, there was no significant displacement of the posterior corneal apex. Individual changes over time did not show a systematic trend across patients, and control subjects experienced changes of the same order of magnitude.

Conclusions. The Pentacam Scheimpflug system can be used reliably to assess changes in the posterior corneal radius of curvature after LASIK. There is no evidence of surgically in-

From the ${ }^{1}$ Instituto de Optica, Daza de Valdés, Consejo Superior de Investigaciones Científicas, Madrid, Spain; and the ${ }^{2}$ Instituto Universitario de Oftalmobiología Aplicada, Universidad de Valladolid, Valladolid, Spain.

Presented in part at the annual meeting of the Association for Research in Vision and Ophthalmology, Fort Lauderdale, Florida, April 2007.

Supported by Project Grants FIS2005-04382 and FIS2008-02065 (Ministerio de Ciencia e Innovación, Spain); Project Grant EURYI-05102-ES (European Heads of Research Councils-European Science Foundation and Ministerio de Ciencia e Innovación); FPU and FPI Predoctoral Fellowships from the Ministerio de Educación y Ciencia; the Consejo Superior de Investigaciones Científicas JAE Program; and the Unidad Asociada Instituto de Optica-CSIC/Instituto de Oftalmobiología Aplicada-Universidad de Valladolid.

Submitted for publication January 14, 2009; revised March 14, 2009; accepted June 15, 2009

Disclosure: A. Pérez-Escudero, None; C. Dorronsoro, None; L. Sawides, None; L. Remón, None; J. Merayo-Lloves, None; S. Mar$\cos$, None

The publication costs of this article were defrayed in part by page charge payment. This article must therefore be marked "advertisement" in accordance with 18 U.S.C. $\$ 1734$ solely to indicate this fact.

Corresponding author: Susana Marcos, Instituto de Óptica Daza de Valdés, Consejo Superior de Investigaciones Científicas, Serrano 121, 28006 Madrid, Spain; susana@io.cfmac.csic.es. duced changes in the corneal posterior surface beyond 1 week after surgery. (Invest Ophthalmol Vis Sci. 2009;50:4146-4154) DOI:10.1167/iovs.09-3411

$\mathrm{C}$ orneal laser in situ keratomileusis (LASIK) intends to change the shape of the anterior surface of the cornea, thus changing its refractive power. ${ }^{1}$ The technique successfully eliminates refractive errors, but high-order aberrations are generally induced by the procedure. ${ }^{2,3}$ The most recent ablation algorithms (wavefront-guided or wavefront-optimized) have been shown to induce fewer high-order aberrations than standard algorithms or even to reduce some high-order aberrations, though discrepancies from the expected optical outcomes are still observed. ${ }^{4,5}$ Refractive outcomes are also subject to some variability because of regression, ${ }^{6}$ hydration, ${ }^{7}$ and other factors that lead to empiric adjustments of the ablation nomogram. ${ }^{8,9}$ Although the surgical procedure does not directly affect the posterior surface of the cornea and a large proportion of the optical changes can be explained at the level of the anterior corneal surface, ${ }^{10}$ a modification in the posterior corneal surface may be expected because the surgery weakens the cornea. Because corneal biomechanical properties are largely unknown, it is not possible to predict theoretically the extent of this potential deformation. ${ }^{11}$

There are some open clinical questions that might be explained by changes on the posterior surface of the cornea. It has been reported that the change in manifest refraction after LASIK differs from the measured change in corneal power, estimated using the standard keratometric index. ${ }^{12}$ Discrepancies may arise from the change in ratio of the anterior/posterior corneal curvature with LASIK (and, therefore, the effective keratometric index), ${ }^{12,13}$ the change in the corneal effective index of refraction caused by epithelial hyperplasia, ${ }^{14}$ or the change in posterior corneal curvature with LASIK. Moreover, theoretical calculations of postoperative corneal shape after subtraction of standard ablation profiles differ dramatically from clinical outcomes. ${ }^{15-18}$ Although it has been demonstrated that, to a large extent, this effect can be explained by physical laws, ${ }^{19,20}$ given that it is also encountered when plastic corneas are ablated, ${ }^{21}$ results are likely affected by the assumption of a mechanically inert cornea. ${ }^{22}$ Empiric corrections of the ablation algorithms can compensate for losses in laser efficiency ${ }^{21}$ and perhaps for systematic deviations found in the average population, but they cannot provide individual adjustments because the reason for the intersubject variability in achieved correction is unknown. ${ }^{11}$ Greater understanding of the biomechanical processes that take place in the cornea after surgery would help to improve the predictability and stability of achieved corrections.

Some previous experimental studies have reported significant changes in the posterior surface of the cornea after LASIK. ${ }^{23-27}$ However, these results have been largely contested because they may be the result of artifacts caused by the optical distortion produced by the anterior surface of the cornea. $^{28,29}$ Recently a Scheimpflug imaging-based commercial device that corrects for this distortion, Pentacam (Oculus $\mathrm{GmbH}$, Wetzlar, Germany), has become available. The first 
studies with this device have reported no significant changes in the posterior surface of the cornea after LASIK and photorefractive keratectomy. ${ }^{30-32}$ Although some studies have reported high repeatability of the Pentacam instrument in nor$\mathrm{mal}^{33}$ and post-LASIK eyes, ${ }^{34}$ the accuracy of this system to measure the posterior cornea had not been validated.

In this study, we validate and use the Pentacam topographer to study changes in the posterior corneal surface after myopic LASIK, comparing them to physiological changes observed in control subjects.

\section{Subjects ANd Methods}

\section{Subjects}

A total of 45 eyes (23 subjects) were used in the study. Fourteen subjects ( 27 eyes), whose ages ranged from 21 to 47 years (32 \pm 7 years [mean $\pm \mathrm{SD}]$ ), underwent refractive LASIK for intended myopic spherical corrections ranging from -1.25 to $-8.50 \mathrm{D}(-4.0 \pm 2.0 \mathrm{D})$. Cylinder ranged from 0 to $-2.5 \mathrm{D}(-0.84 \pm 0.7)$. Patients were tested before and at various times after the procedure. Nine subjects (18 eyes), whose ages ranged from 24 to 43 years ( $32.5 \pm 7$ years), did not undergo surgery and acted as control subjects. The control subjects were tested at the Instituto de Óptica Daza de Valdés with the same Pentacam unit used for the patients. Experimental protocols were approved by an institutional review board and met the tenets of the Declaration of Helsinki. All patients signed informed consent.

\section{Surgical Procedure}

We conducted standard LASIK surgery using a narrow-beam, flyingspot excimer laser (Chiron Technolas 217-C equipped with the Plano Scan program; Bausch \& Lomb Surgical, Rochester, NY). This laser has an emission wavelength of $193 \mathrm{~nm}$, a fixed pulse repetition rate of 50 $\mathrm{Hz}$, and a radiance exposure of $120 \mathrm{~mJ} / \mathrm{cm}^{2}$. The procedure was assisted by an eye tracker. Flaps were performed with suction rings of $8.5-\mathrm{mm}$ ( 3 eyes) or $9.5-\mathrm{mm}$ ( 24 eyes) diameter. We used a microkeratome (Hansatome; Bausch \& Lomb) programmed for 160- $\mu \mathrm{m}$ (22 eyes) or $180-\mu \mathrm{m}$ ( 5 eyes) depth. Photoablation was applied to an optical zone of diameter ranging from 5.2 to $7 \mathrm{~mm}(6.6 \pm 0.6 \mathrm{~mm})$. The LASIK procedures were conducted at the Instituto de Oftalmobiología Aplicada, Universidad de Valladolid, Spain.

Preoperative central pachymetry (measured by Pentacam) ranged from 510 to $643 \mu \mathrm{m}(572 \pm 33 \mu \mathrm{m})$. Residual bed thickness 1 day after surgery (computed as central pachymetry 1 day after surgery minus programmed flap depth) ranged between $271 \mu \mathrm{m}$ and $421 \mu \mathrm{m}$ (329 \pm $41 \mu \mathrm{m})$.

\section{Pentacam Scheimpflug Imaging}

We used a Pentacam (Oculus $\mathrm{GmbH}$ ) anterior segment imaging system based on the Scheimpflug principle. The Scheimpflug camera is a modification of a slit-lamp camera, with a modified geometry to improve depth of focus. The slit rotates around the optical axis of the instrument, allowing the capture of 25 anterior segment diametral sections at different angles; hence, three-dimensional elevation maps of the anterior and posterior corneal surfaces are obtained. According to the manufacturer, this device provides quantitative information of the posterior cornea, as it automatically corrects for optical distortions caused by refraction in the anterior corneal surface. In a previous study, we presented optical and geometrical distortion correction algorithms to obtain quantitative anterior segment biometry and cornea and crystalline lens geometry directly from Pentacam Scheimpflug images $^{35}$ and showed that their application improves the accuracy of the posterior corneal radius of curvature by $30 \%$. The commercial software provides measurements of anterior and posterior radii of curvature (by fitting a best sphere) or measurements of horizontal and vertical apical radii, asphericity, and astigmatism (by fitting a biconic surface). Raw elevation maps are also provided that can be used for further quantitative analysis (Matlab; MathWorks, Natick, MA).

\section{Hybrid Porcine-Plastic Eye Model for Experimental Validation of the Scheimpflug Systems}

Validation of the accuracy of the measurements of the posterior corneal surface geometry was performed in vitro using a hybrid porcine/ plastic eye model. Corneal tissue was used to achieve similar intracorneal scattering in the images, which appears to be critical to achieve good edge detection, and a similar index of refraction, which is a parameter in the reconstruction algorithms.

The hybrid porcine/plastic eye models consisted of excised porcine corneas mounted on a 12-mm-diameter plastic piston finished on a spherical surface. Figure 1 shows a schematic diagram of the model and mount. A strip of sclera was left around the cornea. Corneal samples were fixed on a custom-designed support, using an annular metallic ring that pressed the scleral strip. The piston (hydrated with hyaluronic acid) was slid inside the supporting piece until the corneal button was fit on the spherical surface. Because of scattering properties, Pentacam detects the back surface of the cornea and not the underlying plastic support. This mount was designed to avoid bubbles, folds, and creases of the corneal tissue by achieving a smooth corneal back surface conformed to the plastic surface. The amount of stress produced by the piston affected the anterior corneal surface geometry. Enucleated eyes were obtained in a local slaughterhouse, and the procedures were performed within 4 hours of death.

Several plastic surfaces (acrylic material) of known radii of curvature $(7.47,7.93$, and $8.75 \mathrm{~mm}$ ) were used as support for the posterior corneas in this validation study. The plastic surfaces were polished in a precision optics lathe. Radii of curvature of the sphere were measured using a microscope noncontact profilometer (PL $\mu$; Sensofar, Barcelona, Spain) that also reported negligible deviations from the spherical shape.

Given that accurate reconstruction of the posterior corneal surface relies on accurate measurements of the anterior corneal surface and optical and geometrical distortion reconstruction method, validation of the anterior corneal surface was also performed using high-quality optical glass surfaces (optical calipers with nominal radii of 9.65, 8.00 and $6.15 \mathrm{~mm}$ ).

\section{Measurements: Validation on Model Eyes}

Glass spherical surfaces acting as anterior corneas were measured on the Pentacam system. Ten measurements were conducted on each surface. One of the plastic spherical surfaces used in the hybrid porcine/plastic eye model $(7.93 \mathrm{~mm})$ was also measured directly (acting as the anterior surface).

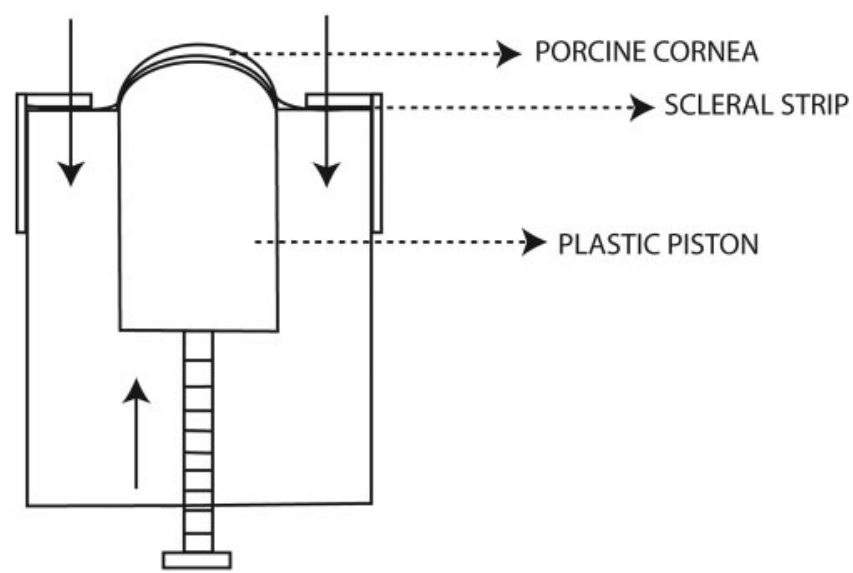

Figure 1. Schematic of the hybrid porcine-plastic eye model for validation of Pentacam. 
Twelve porcine corneas were used in the validation study $(5$ porcine corneas were mounted on $7.47-\mathrm{mm}$ and $8.75-\mathrm{mm}$ spherical corneas, and 2 were mounted on the $7.93-\mathrm{mm}$ spherical cornea). Each hybrid eye was measured up to five times on the Pentacam (total of 36 measurements) using procedures identical to those used in the glass/ plastic eyes and patients. The piston pressure was slightly incremented between every two measurements. Ten measurements (including the whole set of measurements from one porcine cornea) that showed evidence of corneal opacification, edema, or bubble formation either from visual inspection or on the Pentacam were discarded.

Data of anterior and posterior radius of curvature (from the best-fitting sphere or mean horizontal and vertical radii of curvature), asphericity $Q$ (defined as $p-1$ ), and astigmatism were obtained from the Pentacam software. Anterior and posterior corneal elevation maps were also used in custom-developed fitting routines (Matlab; MathWorks).

\section{Measurements: Patients and Control Eyes}

We collected topographies of both eyes of each patient before and several times after surgery. Thirteen eyes were measured at three different points after surgery $-1.3 \pm 0.5,10.5 \pm 3.2$, and $33.4 \pm 5.5$ days-that we will refer as 1 day, 1 week, and 1 month after surgery. Another eye was measured postoperatively only 1 day after surgery, six eyes were measured 1 week after surgery, two eyes were measured 1 week and 1 month after surgery, and five eyes were measured 1 month after surgery. Average measurement times for the whole group of eyes were $1.3,10.8$, and 33.4 days. In control subjects, we acquired several consecutive topographies of both eyes separated by 1 day and 1 week. These data were used as baseline for normal short-term and midterm changes in the posterior corneal shape. Time of the measurement across subjects varied between 9:00 am and 4:00 pm, but for each subject measurements were conducted around the same time of day (within $3.4 \pm 1.4$ hours). We acquired three to six valid topographies per session. One of the control eyes was measured 23 consecutive times to study instrument repeatability.

\section{Data Analysis}

Surface Fitting. We exported topographic maps of anterior and posterior corneal surfaces from Pentacam (Fig. 2A) and used technical computing software (Matlab; MathWorks) for the data analysis to have greater freedom with data manipulation than permitted by the Pentacam software and to ensure that the definitions of apical radius of curvature and asphericity complied with known equations. We computed apical radii and asphericity by fitting the central $6 \mathrm{~mm}$ of the corneal elevation maps to bi-elliptical surfaces (ellipsoids). Unless clearly stated otherwise, the data presented in this article will be referred to as rotationally symmetric ellipsoid.

Computing software (Matlab; MathWorks) fitting routines could not be used in all the hybrid eyes because the exporting procedure is interrupted by the software when no iris is detected. Therefore, for these instances, we used the Pentacam fitting routines. We studied the agreement between the radii of curvature and the asphericity obtained by our fitting procedure and those obtained by the Pentacam software.

Significance of Changes. The repeatability of the instrument limits the significance of the changes found in the measurements and the conclusions that can be extracted. We present the average of repeated measurements and used a Student's $t$-test to assess the significance of the changes across days (at a significance level of $P<0.017$ after application of the Bonferroni correction).

Difference Maps and Corneal Elevation. We subtracted preoperative and postoperative posterior corneal elevation maps to obtain difference elevation maps (see Fig. 2B). Proper alignment of these surfaces is critical to prevent the misalignment artifacts from affecting the difference maps. In principle, we should align the topographies both in axis (rotation) and in position (translation). We found that the raw topographies were already aligned in axis as we performed the analysis both with and without rotation in the alignment, ${ }^{35}$ and we did not observe statistically significant differences between the results obtained with either
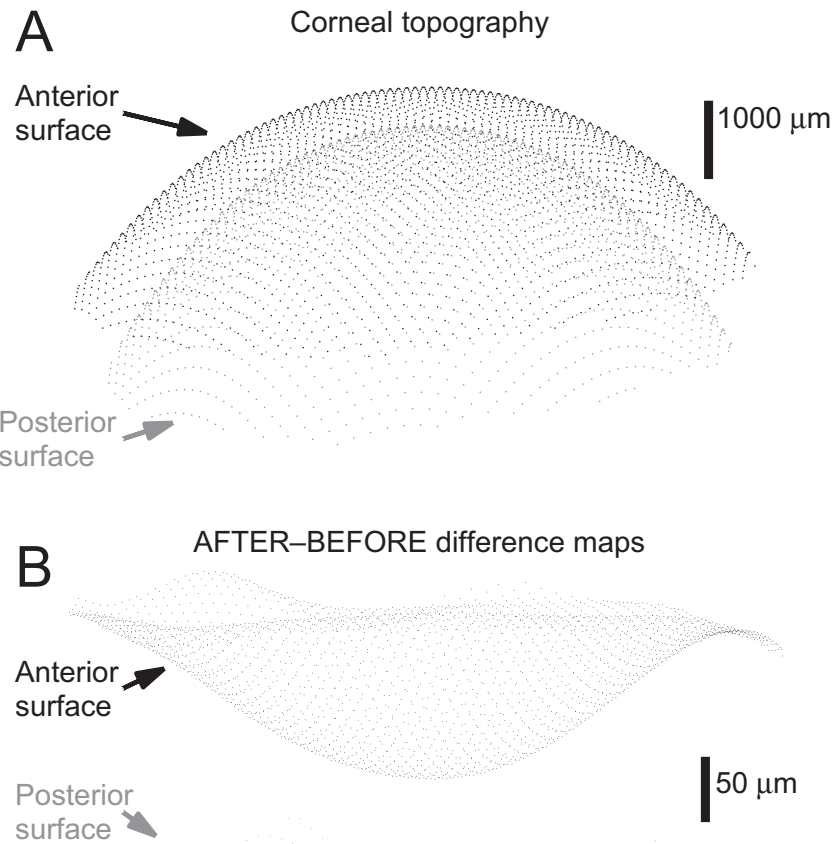

Figure 2. (A) Elevation data exported from Pentacam. Each corneal surface is represented by a cloud of points arranged in an $x-y$ fashion on a $100 \times 100-\mu \mathrm{m}$ square grid. The distance between both corneal surfaces reflects the central pachymetry measured by Pentacam. (B) Difference maps, obtained after alignment using custom routines (Matlab; MathWorks). Distance between anterior and posterior difference maps is arbitrary, chosen for optimal visualization.

method. To align the two measurements in position, we used the fit without rotation to achieve the alignment in the $X-Y$ plane (where $Z$ is the line of sight). Thus, we translated both topographies so that the center of their best-fit ellipsoids fell in the origin. Then, we translated the postoperative measurement in $Z$ until the differences between the topographies were minimal. For a study of repeatability of Pentacam, we used the whole surface for alignment. For computation of longitudinal displacement of posterior corneal apex, we used the peripheral ring between 6 and 10-mm diameters for alignment of both topographies. Then, we computed the apex longitudinal displacement as the difference between elevations of the posterior corneal surface apex after surgery minus elevation of the posterior corneal apex before surgery (a forward bulging corresponds to a positive number). In this article, we call corneal apex the point where the principal axis of the best-fit ellipsoid intersects with the corneal surface.

\section{Results}

\section{Validation of the Pentacam Scheimpflug Imaging System on a Hybrid Eye}

Validation of the Anterior Surface. Table 1 shows the nominal and measured radii of curvature of glass spherical models. Anterior radii of curvature were estimated within an accuracy of $0.04 \mathrm{~mm}$ with respect to the nominal values of the reference spheres (typically slightly underestimated). Asphericity was $-0.02 \pm 0.22$ on average, close to the expected value (zero) for a sphere, though the variability was high. Astigmatism was higher than expected (power $0.32 \pm 0.27$, on average) but with random angle, indicating that there are not predominant orientations and that the average surface is a 
TABLE 1. Validation of Pentacam Measurements for Anterior Radius

\begin{tabular}{cccc}
\hline $\begin{array}{c}\text { Glass } \\
\text { Sphere }\end{array}$ & $\begin{array}{c}\text { Nominal R } \\
(\mathbf{m m})\end{array}$ & $\begin{array}{c}\text { Measured R } \\
\text { (anterior surface) } \\
(\mathbf{m m})\end{array}$ & $\begin{array}{c}\text { Difference } \\
\text { (mm) }\end{array}$ \\
\hline 1 & 9.65 & $9.61 \pm 0.04$ & 0.04 \\
2 & 8.00 & $7.97 \pm 0.01$ & 0.03 \\
3 & 6.15 & $6.12 \pm 0.02$ & 0.03 \\
\hline
\end{tabular}

sphere, as expected. The repeated measurements on the 7.93-mm plastic surface provided similar results, but the cylindrical geometry of the piston produced unwanted reflections that prevented systematic measurements in all samples.

Validation of the Posterior Surface. Table 2 shows the nominal and estimated posterior radii of curvature in the hybrid porcine/plastic eye model. Each datum is the average of the different eyes with similar posterior plastic cornea. Correlation is good between nominal and estimated radius, but bias (underestimation) is higher than for the anterior cornea $(150-400 \mu \mathrm{m})$. Despite the low accuracy (the absolute values are not recovered exactly), the repeatability is good (below $100 \mu \mathrm{m}$ ) considering that these are individual measurements (nonaveraged) of hybrid eyes whose anterior surface varies across measurements.

Correlations between anterior and posterior corneal surfaces were performed to assess that the optical distortion correction was successfully applied. Statistically significant correlations between the posterior (fixed) and the anterior (variable, as dependent from the specimen and pressure exerted by the piston) corneal surfaces would be indicative of artifacts in the correction. This is particularly critical in the present study, when the same eyes were evaluated before and after a change in the anterior cornea.

Our hybrid porcine/plastic model corneas showed a wide range of anterior radii (from 8.0 to $9.6 \mathrm{~mm}$ ) for the different corneas. A $z$-test showed no correlation between anterior and posterior corneal radius for any of the eyes $(P>0.6)$, indicating that the anterior surface did not affect the measurement of the posterior surface. In addition, the anterior corneas typically showed significant amounts of astigmatism $(1.9 \pm 1.0 \mathrm{D})$ with preferential orientations in the vertical and horizontal directions, whereas the estimated astigmatism for the posterior surface was much lower $(0.31 \pm 0.15 \mathrm{D})$, with no preferential orientation. These retrieved values of astigmatism of the posterior corneal surfaces conformed to spherical plastic surfaces and were similar to those found in the calibrations of spherical model eyes (anterior surfaces) in the previous section. There was no correlation between the astigmatism of the anterior and posterior corneal surfaces ( $P=0.57$ for all eyes). Finally, there was no correlation between the anterior and posterior corneal asphericities ( $P=0.28$ for all the eyes). However, the posterior corneal asphericity differed from zero and was variable $(-0.33 \pm 0.44)$.

\section{Repeatability of the Posterior Corneal Surface Measurements on Control Eyes}

To estimate the repeatability of the Pentacam, we measured the eye of one control subject 23 consecutive times. These

TABLE 2. Validation of Pentacam Measurements for Posterior Radius

\begin{tabular}{cccc}
\hline $\begin{array}{c}\text { Plastic } \\
\text { Sphere }\end{array}$ & $\begin{array}{c}\text { Nominal R } \\
(\mathbf{m m})\end{array}$ & $\begin{array}{c}\text { Measured R } \\
\text { (posterior surface) } \\
(\mathbf{m m})\end{array}$ & $\begin{array}{c}\text { Difference } \\
\mathbf{( m m )}\end{array}$ \\
\hline 1 & 7.47 & $7.32 \pm 0.1$ & 0.15 \\
2 & 7.93 & $7.54 \pm 0.05$ & 0.39 \\
3 & 8.75 & $8.51 \pm 0.08$ & 0.24 \\
\hline
\end{tabular}

measurements took less than 30 minutes, so any change should be attributed to short-term dynamic ocular variations and, primarily, measurement errors. We considered the dispersion of these measurements as an estimate of the repeatability of Pentacam in real eyes. We computed the standard deviation of each point of the difference map after aligning the 23 posterior corneal surface topographies, as described, using the whole topography for alignment. These deviations are shown in Figure 3. In the periphery of the topographic map, the standard deviation is as high as $40 \mu \mathrm{m}$. By restricting the analysis to the 6-mm diameter central area, we ensure that standard deviation is below $10 \mu \mathrm{m}$. We also used these 23 measurements to estimate the dispersion of the radii of curvature and asphericities of the anterior and posterior corneal surfaces. The standard deviation for the anterior radius was $10 \mu \mathrm{m}$, and it was 0.03 for the anterior asphericity. We found a higher standard deviation for the posterior radius, $\sigma_{\mathrm{R}}=25 \mu \mathrm{m}(R=6.2 \mathrm{~mm}$ the average radius for this eye) and posterior asphericity of $\sigma_{\mathrm{Q}}=0.06(Q=$ 0.11 the average asphericity for this eye). We obtained the same results with both processing methods (Pentacam processing and our fits to central $6 \mathrm{~mm}$ ).

\section{Comparison of Software-Fitting Parameters}

We studied whether Matlab and Pentacam provided similar estimates of radius of curvature and asphericity on 18 control eyes (3-5 repeated measurements on each eye). We found a mean difference between methods of $10 \mu \mathrm{m}$ in the anterior radius of curvature and of $-16 \mu \mathrm{m}$ in the posterior radius of curvature, a mean difference of -0.04 in the asphericity of both surfaces. These values are below the standard deviation of repeated measurements from each method.

\section{Change of the Anterior Corneal Surface with LASIK Surgery}

Although this study focuses on the posterior corneal surface, we checked that the anterior corneal measurements obtained using Pentacam agree with those previously reported using other methods. We observed a statistically significant increase of both anterior radius and asphericity in all postoperative measurements. The first day after surgery, the change in radius ranged from $268 \mu \mathrm{m}$ to $1608 \mu \mathrm{m}(674 \pm 348 \mu \mathrm{m}, P<0.0001)$, and the change in asphericity $(Q)$ ranged from 0.07 to $1.88(0.55 \pm 0.47, P<$

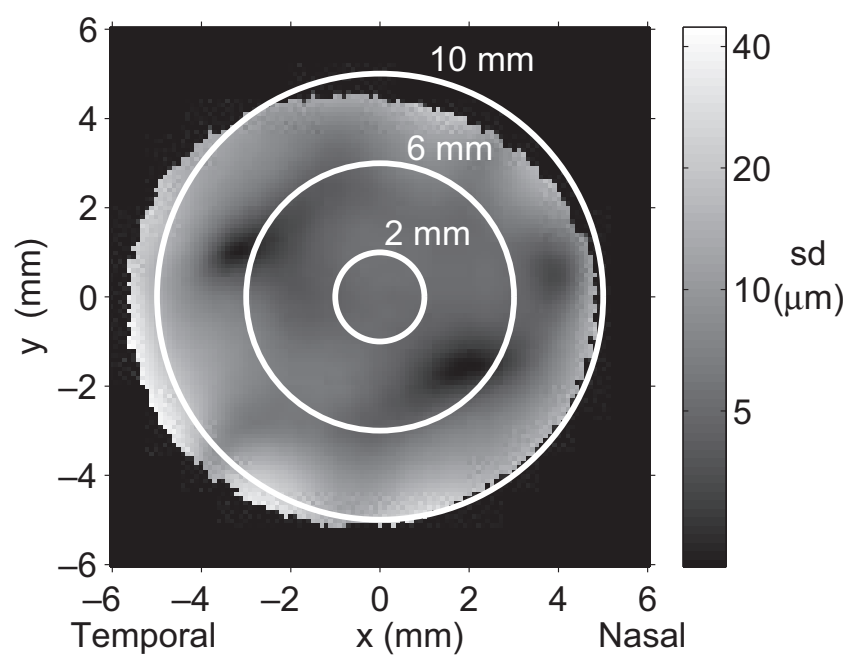

Figure 3. Standard deviation of Pentacam posterior corneal surface elevation maps $(\mu \mathrm{m})$. White circles limit central areas with specified diameters. Analysis was made on the 6-mm diameter, where standard deviation is below $10 \mu \mathrm{m}$. 
0.0001). We found high correlations between change in radius and attempted correction $(r=0.94, P<0.0001)$ and between change in asphericity and attempted correction $(r=0.82, P<$ $0.0001)$. These results are consistent with previous reports of changes in the anterior corneal surface after LASIK with the same laser used for the present study. ${ }^{15}$

\section{Change of the Posterior Corneal Surface as a Function of Time}

Table 3 shows the average radii and asphericities of both corneal surfaces of all patients. We studied the evolution over time of the change in the posterior corneal surface (postoperative minus preoperative values) for the 13 eyes that were measured at all time points. Results are shown for average change of the radius of curvature of the posterior surface of the cornea in Figure 4A, gray dots, and for average change in asphericity of the posterior corneal surface in Figure $4 \mathrm{~B}$, gray dots. Error bars limit the $98.3 \%$ (corresponding to $\alpha=0.05 / 3$, according to Bonferroni correction) confidence interval given by Student's $t$ distribution. This population of eyes shows a near-significant decrease radius $(\Delta R=-29 \mu \mathrm{m}, P=0.06)$ and a statistically significant decrease of asphericity 1 day after surgery $(\Delta Q=-0.08, P=0.005)$. No statistically significant changes are present 1 week or 1 month after surgery. When the entire population of eyes is included (Fig. 4, black squares), we observe a near-significant decrease of radius $(\Delta R=-28$ $\mu \mathrm{m}, P=0.05)$ and a statistically significant decrease of asphericity $(\Delta Q=-0.06, P=0.01)$ the first day after surgery and no statistically significant change 1 week and 1 month after surgery. Figure $4 \mathrm{C}$ shows the measured longitudinal displacements of the apex of the posterior corneal surface. These displacements were less than $35 \mu \mathrm{m}$ in individual eyes, and on average they were not statistically significant at any time point.

Figure 5 shows individual changes in radius of curvature versus time for the six patients whose eyes both underwent the three measurements ( 1 day, 1 week, and 1 month). Each panel shows data on a single subject. Solid lines represent right eyes, and dotted lines represent left eyes. Points marked with a filled dot mean a statistically significant change with respect to the preoperative measurement, whereas empty circles mean that the change is not statistically significant (two-sample unpaired Student's $t$-test between the repeated preoperative and postoperative measurements; $\alpha=0.05 / 3$ ). No systematic trend was followed by all patients, but statistically significant changes were frequent. Four eyes showed statistically significant and sustained decreases in posterior radius after surgery (Figs. 5A, B, E). On the other hand, two eyes showed the opposite: systematic and sustained increases in radius (Figs. 5D, F). Most patients showed nonsystematic behavior.

We compared the changes in eyes of patients who underwent refractive surgery (Figs. 5A-F) with those of normal control eyes measured after a similar time course (Figs. 5G-L). In some cases, we observed statistically significant changes in control eyes between measurements separated 1 day or 1 week, although no treatment had been applied between the

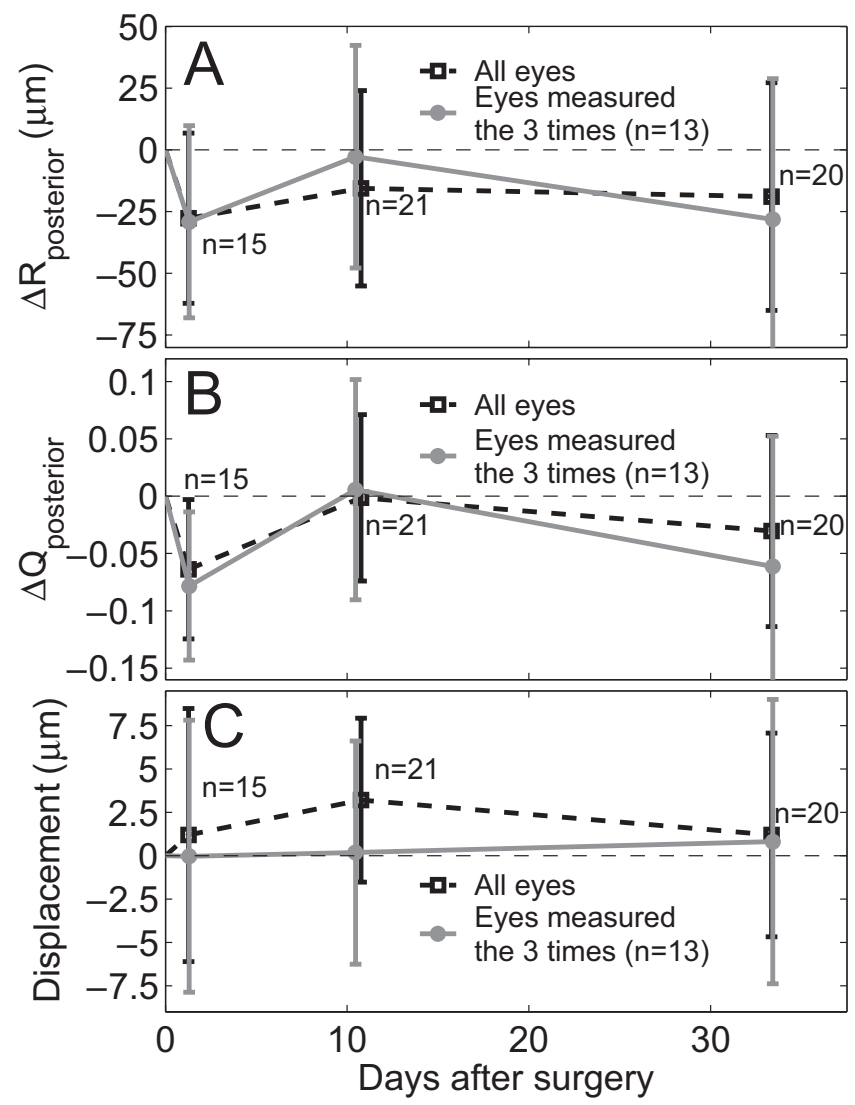

Figure 4. (A) Change in the apical radius of curvature of the posterior corneal surface versus time. Error bars limit the $98.3 \%$ confidence interval. Black squares: all eyes (each square comes from averaging a different subset of eyes; the number of eyes in each subset is shown in the legends near the squares). Gray dots: eyes for which all three measurements were available. Each dot represents an average across 13 eyes. A positive sign is indicative of posterior corneal flattening, and a negative sign is indicative of posterior corneal steepening. (B) Change in the average asphericity of the posterior corneal surface versus time. A positive sign indicates changes toward oblateness, and a negative sign indicates changes toward prolateness. (C) Change in the elevation of the apex of the posterior corneal surface. A positive sign indicates posterior forward bulging, and a negative sign indicates backward bulging.

measurements. Interestingly, left and right eyes of the same subject (in all patients and all but one control subject) followed the same trends with time.

\section{Difference between Vertical and Horizontal Radii and Asphericities}

The results reported above correspond to radii and asphericities obtained from a fit of the topographies to rotationally

TABLe 3. Average Values for All Patients

\begin{tabular}{|c|c|c|c|c|}
\hline & \multicolumn{2}{|c|}{ Anterior Corneal Surface } & \multicolumn{2}{|c|}{ Posterior Corneal Surface } \\
\hline & Radius ( $\mu \mathrm{m})$ & Asphericity & Radius ( $\mu \mathrm{m})$ & Asphericity \\
\hline Before surgery & $7700 \pm 240$ & $-0.10 \pm 0.12$ & $6380 \pm 220$ & $0.16 \pm 0.24$ \\
\hline 1 day after surgery & $8430 \pm 430$ & $0.42 \pm 0.50$ & $6420 \pm 210$ & $0.06 \pm 0.28$ \\
\hline 1 week after surgery & $8460 \pm 480$ & $0.42 \pm 0.53$ & $6400 \pm 180$ & $0.19 \pm 0.28$ \\
\hline 1 month after surgery & $8520 \pm 390$ & $0.59 \pm 0.53$ & $6370 \pm 220$ & $0.12 \pm 0.26$ \\
\hline
\end{tabular}

All data are displayed as mean \pm SD. 


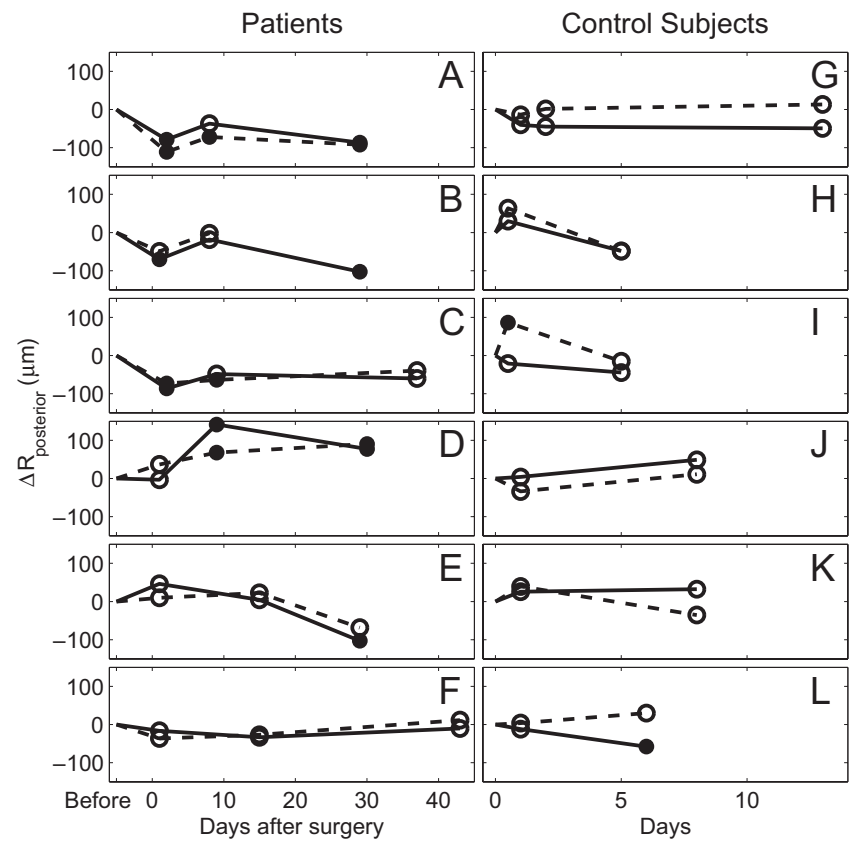

FIGURE 5. Changes in postoperative radii of curvature of the posterior corneal radius relative to the preoperative value. Each panel shows data for one patient. Solid line: right eye. Dashed line: left eye. Filled dots: statistically significant changes $(\alpha=0.017)$. (A-F) Patients whose eyes were measured three times after surgery (except the left eye of patient $\mathrm{B}$, for whom fewer than three valid topographies were available 1-month after surgery). (G-L) Controls whose eyes were measured all three times.

symmetric ellipsoids. We have also fitted the topographies to more general nonrotationally symmetric ellipsoids and analyzed the vertical and horizontal radii and asphericities separately. We did not find statistically significant meridional differences in asphericity (Figs. 6C, D). However, the average radius of curvature showed statistically significant meridional differences: although the horizontal radius did not change at any point (Fig. 6A), the vertical radius of curvature underwent a statistically significant change 1 day after surgery (Fig. 6B; $\left.\Delta R_{y}=47 \mu \mathrm{m} ; P=0.004\right)$. This meridional difference was equivalent to a posterior astigmatism of $0.9 \mathrm{D}$ (and an effective astigmatism of $0.037 \mathrm{D})$ and was responsible for the nearsignificant change of the average radius of curvature reported previously (Fig. 4A). This meridional change could not be attributed to different variability in the vertical direction for this instrument, as seen from the validation experiments. No meridional differences were found in control subjects.

Figure 7 shows histograms of changes between preoperative and all postoperative measurements for all control eyes (A, B) and all eyes that underwent $(C, D)$. Changes in control subjects followed normal distributions (according to JarqueBera normality test) for both meridians (Figs. 7A, B). Both distributions have zero mean (Student's $t$-test), and although there is more dispersion for the vertical meridian, there is no statistically significant difference between the two standard deviations $(P=0.06$; Ansari-Bradley test). We conclude that there was no statistically significant difference between horizontal and vertical radius of curvature for control subjects. Changes in patients were not normally distributed (Figs. 7C, D), and their standard deviations were slightly greater than the standard deviations of changes in controls for both meridians. We observed no statistically significant shift for the horizontal radius (Fig. 7 C), but there was a significant shift of the vertical radius 1 day after surgery that disappeared afterward (Fig. 7D).

\section{Correlation of the Posterior Corneal Changes with Different Parameters}

We tested correlations between posterior corneal changes in radius of curvature, asphericity, or central elevation with the attempted correction, change of pachymetry, preoperative central pachymetry, postoperative central pachymetry, or postoperative residual bed thickness. We tested all these correlations separately for changes 1 day, 1 week, and 1 month after surgery. Of those correlations, only the correlation between attempted correction and posterior corneal apex shift 1 week after surgery showed a statistically significant correlation $(r=$ $0.665 ; P=0.0005)$. We found near-significant correlations $(P=0.04-0.051)$ only between attempted correction and posterior corneal apex shift 1 day after surgery, and pachymetry, posterior apex shift, and radius of the posterior surface 1 week after surgery. These correlations disappear if eyes with attempted corrections greater than $-6 \mathrm{D}$ are removed from the
FIGURE 6. Changes in average horizontal and vertical radii of curvature (A, B) and asphericity (C, D). Gray dots: patients whose eyes were measured three times after surgery. Black squares: all patients. Error bars indicate the $98.3 \%$ confidence interval. Number of eyes per point is the same as in Figure 4.

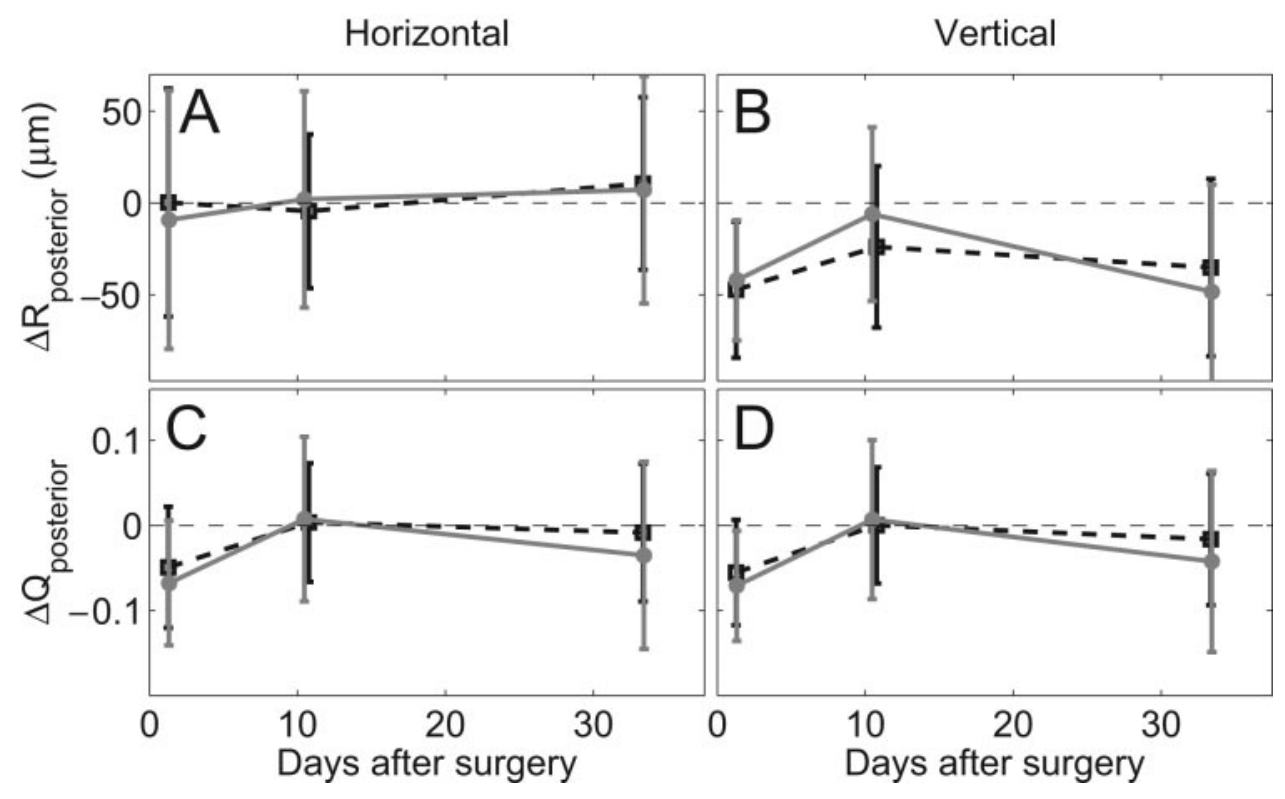


Horizontal
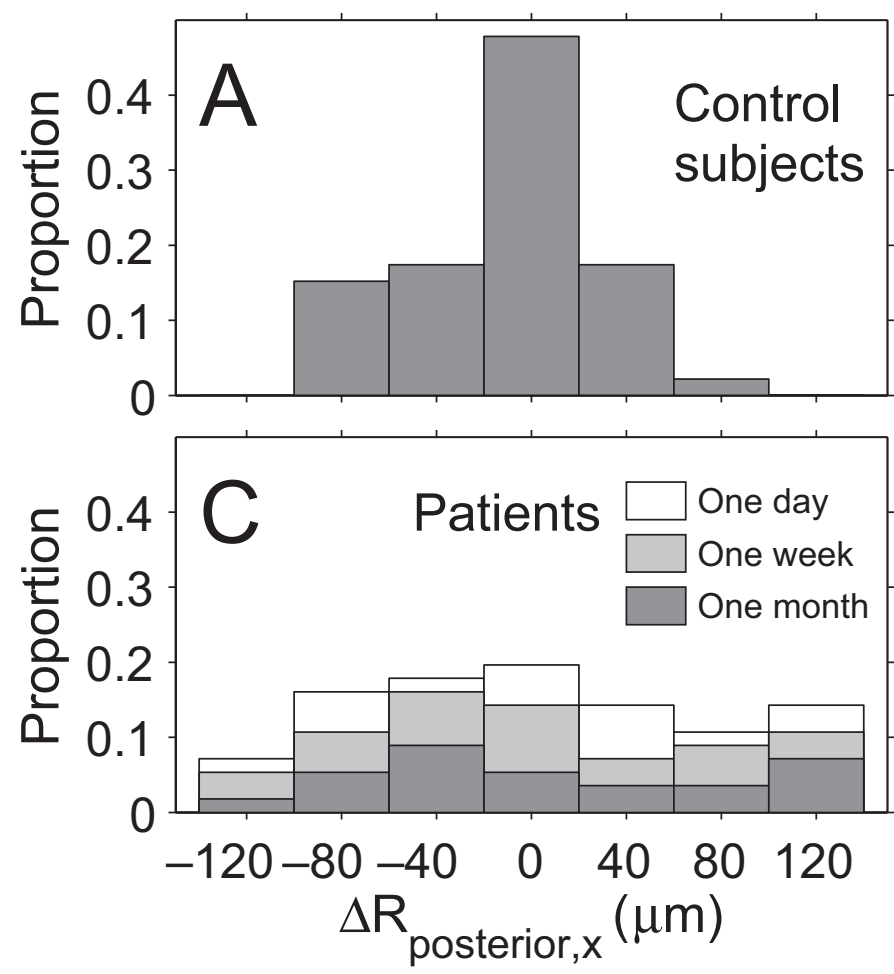

Vertical
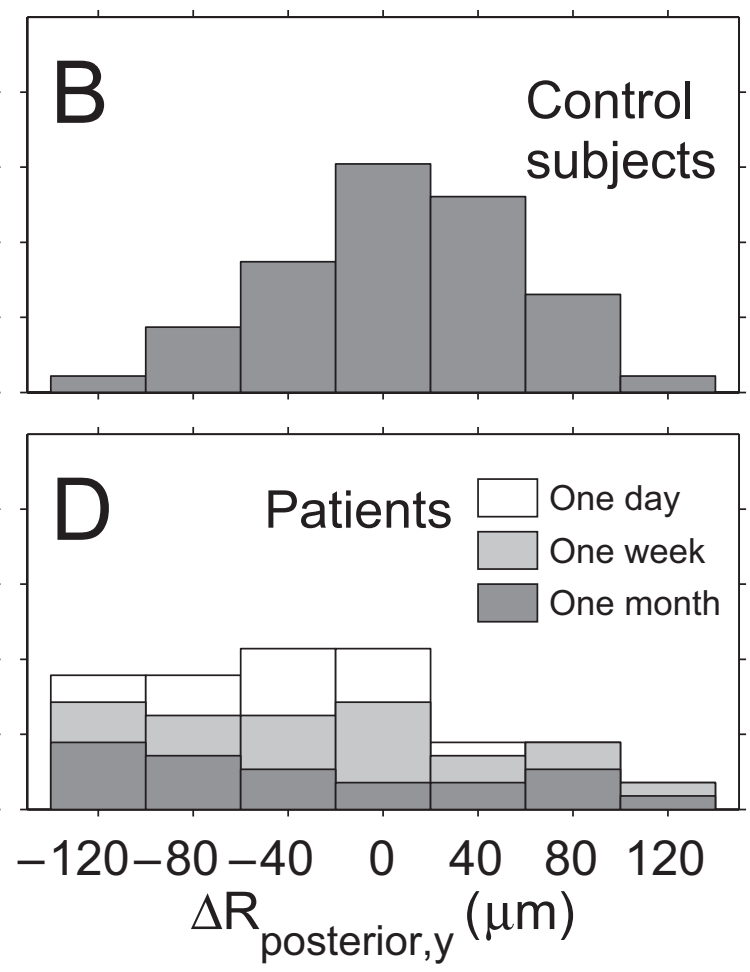

FIGURE 7. (A) Histogram of changes in horizontal radius of curvature of the posterior corneal surface in all control subjects, including all changes at 1 day and 1 week. (B) Histogram of changes in vertical radius of curvature of the posterior corneal surface in all control subjects, including all changes at 1 day and 1 week. (C) Histogram of changes in horizontal radius of curvature of the posterior corneal surface in all patients, between preoperative measurement and all postoperative measurements (1 day, 1 week, and 1 month). (D) Histogram of changes in vertical radius of curvature of the posterior corneal surface in all patients, between preoperative measurement and all postoperative measurements (1 day, 1 week, and 1 month).

calculation. There were no statistically significant correlations 1 month after surgery.

\section{Discussion}

We have presented a validation of the Pentacam Scheimpflug system to assess the posterior corneal surface geometry and measurements of changes in the posterior corneal surface in patients who underwent myopic LASIK refractive surgery and in control subjects.

The validation experiments using a hybrid porcine/plastic eye model indicate that the posterior corneal radius can be obtained with high repeatability and that the reconstructed posterior surface is not affected by changes or asymmetries in the anterior cornea. Posterior corneal radius seems to be slightly underestimated and asphericity overestimated in comparison with the nominal values of the posterior cornea in the model eye used for calibration. Although studies on the repeatability of this system had been published (particularly of the anterior radius of curvature and pachymetry $\left.{ }^{33,34}\right)$, this is, to our knowledge, the first time the accuracy (for both anterior and posterior surfaces) has been studied.

Our results for anterior surface mean preoperative radius $\left(R_{\text {ant }}=7740 \pm 230 \mu \mathrm{m}\right)$ and asphericity $\left(Q_{\mathrm{ant}}=-0.11 \pm\right.$ 0.13 ) in our group of patients agree well with those reported by Dubbelman et al., ${ }^{36}$ who used a custom-adapted Scheimpflug imaging system $\left(R_{\text {ant }}=7870 \pm 270 \mu \mathrm{m} ; Q_{\text {ant }}=-0.18 \pm\right.$ $0.18) .{ }^{1}$ There is also good agreement between our mean preoperative posterior corneal radius of curvature $\left(R_{\mathrm{back}}=\right.$ $6440 \pm 250 \mu \mathrm{m})$ and that of Dubbelman et al. ${ }^{36}\left(R_{\text {back }}=\right.$
$6400 \pm 280 \mu \mathrm{m})$. However, our mean preoperative posterior corneal asphericity was significantly higher $\left(Q_{\text {back }}=0.18 \pm\right.$ 0.21 in our study and $Q_{\text {back }}=-0.38 \pm 0.27$ in Dubbelman et al. ${ }^{36}$ for $6-$ and $7-\mathrm{mm}$ fitted areas, respectively). Differences might have arisen from differences in the refractive state and age of both populations because our results from the validation using a hybrid model eye indicate an underestimation rather than an overestimation of the posterior corneal asphericity.

We have not found evidence of a systematic influence of LASIK on the posterior surface of the cornea on average. We detected a change in the radius of curvature and asphericity of the posterior corneal surface the first day after surgery, but afterward this change disappeared. The cause of this change is unclear to us. The fact that changes disappeared in days to weeks suggests the action of biological processes within that time. Potential factors affecting posterior corneal shape temporarily may include hydration, keratocyte activity, stress produced by the suction ring of the microkeratome, or medication. Furthermore, we observed a remarkable similarity between both eyes of each subject, suggesting a physiological mechanism acting bilaterally in the same way because both corneas of the same patient are expected to have similar biomechanical properties and to follow similar biological processes. Bilateral similarity also tended to occur in left and right eyes of the control subjects.

We have found that changes in the posterior radius of curvature occur primarily in the vertical direction in patients after LASIK. There are at least three possible causes for this effect. First, it is possible that surgery affects corneal stability more strongly in the vertical direction possibly because of an 
asymmetrical ablation for correction of astigmatism, but we did not find any statistically significant correlation between corrected astigmatism and radius change. Even with symmetric ablation corneal stability could be more strongly affected in the vertical direction because of the direction of the flap, which is cut in the vertical direction, leaving a superior hinge. Second, meridian-independent surgery may cause a greater change in the vertical meridian if there is a greater mechanical stress of the cornea in that direction. This higher mechanical stress may be caused by the eyelid, which presses on the superior part of the cornea and has been shown to modify the corneal geometry and to have an impact on corneal aberrations. ${ }^{37}$ Third, intralamellar cohesive strength, studied in human eye bank corneas, has been shown to be lower in the vertical than in the horizontal meridian, suggesting that even a symmetric force applied to the cornea may result in an asymmetric corneal deformation. ${ }^{38}$ In previous studies, ${ }^{34}$ the vertical posterior radius of curvature was found to be the least repeatable of the parameters measured by Pentacam, in repeated measurements of patients after LASIK.

Control subjects also experienced statistically significant changes of the same order of magnitude as found in patients, though the reported differences between vertical and horizontal meridians were unique to patients (see Fig. 7D; see also Fig. 6B). These differences between patients and control subjects were indicative of some surgical effect on the posterior corneal surface, though the average magnitude of the changes observed in patients was similar to that observed in control subjects. This suggested that most of the changes observed in patients were normal, perhaps because of changes in intraocular pressure. In fact, preliminary results of inflation experiments on porcine eyes show that the radius of curvature of the posterior corneal surface changes approximately $30 \mu \mathrm{m} / \mathrm{mm} \mathrm{Hg}$ (Pérez-Escudero A, et al. IOVS 2008;49:ARVO Abstract 664). The changes in posterior radius of curvature that we report in the present study (up to 120 $\mu \mathrm{m})$ are consistent with changes of intraocular pressure of the order of $5 \mathrm{~mm} \mathrm{Hg}$, which is the average change in intraocular pressure throughout the day. ${ }^{39}$ The small changes induced by the surgery are superposed to these physiological changes and initiate the subtle correlations mentioned.

All changes we found in the radius of curvature of the posterior corneal surface were smaller than $180 \mu \mathrm{m}$ (taking into account an interval of confidence of 98.3\%). In an average cornea, this change in radius induces a change in the refractive power of the posterior corneal surface below $0.18 \mathrm{D},{ }^{40}$ too small to be clinically relevant. Therefore, the contribution of the posterior corneal surface to shifts from the attempted refraction is minor. Previous studies with a topographer (Orbscan; Bausch \& Lomb) reported long-term average changes in posterior radius of curvature up to $400 \mu \mathrm{m},{ }^{24,26}$ and ectasia measured as forward displacement of the center of the posterior corneal surface up to $40 \mu \mathrm{m},{ }^{23,25}$ much greater than the changes observed by us (lower than $8 \mu \mathrm{m}$ on average, including the $98.3 \%$ confidence interval; Fig. 4C). The discrepancy might have resulted from improper correction of the distortion because of the anterior corneal surface in the topographer (Orbscan; Bausch \& Lomb). ${ }^{28,29}$ Our results are consistent with recent data obtained with the same device (Pentacam). ${ }^{30}$ Along with the findings of this study, more experimental data on corneal biomechanical properties and more accurate models of corneal biomechanics will help to better our understanding of the corneal shape response to LASIK surgery.

\section{Acknowledgments}

The authors thank Samuel Arba Mosquera and José Requejo-Isidro for fruitful discussions and comments and Sara Arganda for help with final formatting of the figures.

\section{References}

1. Pallikaris IG, Papatzanaki ME, Stathi EZ, Frenschock O, Georgiadis A. Laser in situ keratomileusis. Lasers Surg Med. 1990;10(5):463468.

2. Applegate RA, Howland HC. Refractive surgery, optical aberrations and visual performance. J Refract Surg. 1997;13(3):295-299.

3. Moreno-Barriuso E, Merayo Lloves J, Marcos S, Navarro R, Llorente L, Barbero S. Ocular aberrations before and after myopic corneal refractive surgery: LASIK-induced changes measured with laser ray tracing. Invest Ophthalmol Vis Sci. 2001;42:1396-1403.

4. Padmanabhan P, Mrochen M, Basuthkar S, Viswanathan D, Joseph R. Wavefront-guided versus wavefront-optimized laser in situ keratomileusis: contralateral comparative study.J Cataract Refract Surg. 2008;34(3):389-397.

5. Kim A, Chuck RS. Wavefront-guided customized corneal ablation. Curr Opin Ophthalmol. 2008;19(4):314-320.

6. Chayet AS, Assil KK, Montes M, Espinosa-Lagana M, Castellanos A, Tsioulias G. Regression and it mechanisms after laser in situ keratomileusis in moderate and high myopia. Ophthalmology. 1998; 105(7):1194-1199.

7. Feltham MH, Stapleton F. The effect of water content on the 193 nm excimer laser ablation. Clin Experiment Ophthalmol. 2002; 30(2):99-103.

8. Mrochen M, Hafezi F, Iseli HP, Löffler J, Seiler T. Nomograms for the improvement of refractive outcomes. Ophthalmolge. 2006; 103(4):331-338.

9. Ditzen K, Handzel A, Pieger S. Laser in situ keratomileusis nomogram development. J Refract Surg. 1999;15(2 suppl):S197-S201.

10. Marcos S, Barbero S, Llorente L, Merayo-Lloves J. Optical response to LASIK surgery for myopia from total and corneal aberration measurements. Invest Ophthalmol Vis Sci. 2001;42(13):3349-3356.

11. Dupps WJ, Wilson SE. Biomechanics and wound healing in the cornea. Exp Eye Res. 2006;83:709-720.

12. Tang M, Li Y, Avila M, Huang D. Measuring total corneal power before and after laser in situ keratomileusis with high-speed optical coherence tomography. J Cataract Refract Surg. 2006;32:1843-1850.

13. Jarade E, Abi Nader F, Tabbara K. Intraocular lens power calculation following LASIK: determination of the new effective index of refraction. J Refract Surg. 2006;22:75-80.

14. Spadea L, Fasciani R, Necozione S, Balestrazzi E. Role of the corneal epithelium in refractive changes following laser in situ keratomileusis for high myopia. J Refract Surg. 2000;16:133-139.

15. Cano D, Barbero S, Marcos S. Comparison of real and computersimulated outcomes of LASIK refractive surgery. J Opt Soc Am A. 2004;21(6):926-936.

16. Marcos S, Cano D, Barbero S. Increase in corneal asphericity after standard laser in situ keratomileusis for myopia is not inherent to the Munnerlyn algorithm. J Refract Surg. 2003;19(5):S592-S596.

17. Gatinel D, Hoang-Xuan T, Azar DT. Determination of corneal asphericity after myopia surgery with the excimer laser: a mathematical model. Invest Ophthalmol Vis Sci. 2001;42(8):1736-1742.

18. Jiménez JR, Anera RG, Jiménez del Barco L. Equation for corneal asphericity after corneal refractive surgery. J Refract Surg. 2003; 19(1):65-69.

19. Mrochen M, Seiler T. Influence of corneal curvature on calculation of ablation patterns used in photorefractive laser surgery. J Refract Surg. 2001;17:584-587.

20. Jiménez JR, Anera RG, Jiménez del Barco L, Hita E. Effect on laserablation algorithms of reflection losses and nonnormal incidence on the anterior cornea. Appl Phys Lett. 2002;81(8):1521-1523.

21. Dorronsoro C, Cano D, Merayo-Lloves J, Marcos S. Experiments on PMMA models to predict the impact of corneal refractive surgery on corneal shape. Opt Exp. 2006;14:6142-6156.

22. Munnerlyn CR, Koons SJ, Marshall J. Photorefractive keratectomy: a technique for laser refractive surgery. J Cataract Refract Surg. 1988; 14:46-52.

23. Wang Z, Chen J, Yang B. Posterior corneal surface topographic changes after laser in situ keratomileusis are related to residual corneal bed thickness. Ophthalmology. 1999;106:406-410.

24. Seitz B, Torres F, Langenbucher A, Behrens A, Suárez E. Posterior corneal curvature changes after myopic laser in situ keratomileusis. Ophthalmology. 2001;108:666-672. 
25. Baek TM, Lee KH, Kagaya F, Tomidokoro A, Amano S, Oshika T. Factors affecting the forward shift of posterior corneal surface after laser in situ keratomileusis. Ophthalmology. 2001;108:317320 .

26. Twa MD, Roberts C, Mahmoud AM, Chang JS. Response of the posterior corneal surface to laser in situ keratomileusis for myopia. J Cataract Refract Surg. 2005;31:61-71.

27. Grzybowski DM, Roberts CJ, Mahmoud AM, Chang JS. Model for nonectatic increase in posterior corneal elevation after ablative procedures. J Cataract Refract Surg. 2005;31:72-81.

28. Ueda T, Nawa $Y$, Masuda $K$, Ishibashi $H$, Hara $Y$, Uozato $H$. Posterior corneal surface changes after hyperopic laser in situ keratomileusis. J Cataract Refract Surg. 2005;31:2084-2087.

29. Seitz BS, Torres F, Langenbucher A, et al. Posterior corneal curvature changes after myopic laser in situ keratomileusis (discussion). Opbthalmology. 2001;108:666-673.

30. Ciolino JB, Belin MW. Changes in the posterior cornea after laser in situ keratomileusis and photorefractive keratectomy. J Cataract Refract Surg. 2006;32:1426-1431.

31. Ciolino J, Khachikian S, Cortese M, Belin M. Long-term stability of the posterior cornea after laser in situ keratomileusis. J Cataract Refract Surg. 2007;33:1366-1370.

32. Matsuda J, Hieda O, Kinoshita S. Comparison of central corneal thickness measurements by Orbscan II and Pentacam after corneal refractive surgery. Jpn J Ophthalmol. 2008;52:245-249.
33. Lackner B, Schmidinger G, Pieh S, Funovics M, Skorpik C. Repeatability and reproducibility of central corneal thickness measurement with Pentacam. Orbscan Ultrasound Opt Vis Sci. 2005;82: 892-899.

34. Jain R, Dilraj G, Grewal SPS. Repeatability of corneal parameters with Pentacam after laser in situ keratomileusis. Ind J Ophthalmol. 2007;55:341-347.

35. Rosales $\mathrm{P}$, Marcos S. Pentacam Scheimpflug quantitative imaging of the crystalline lens and intraocular lens. J Refract Surg. 2009;25: 421- 428 .

36. Dubbelman M, Weeber HA, van der Heijde RGL, Völker-Dieben HJ. Radius and asphericity of the posterior corneal surface determined by corrected Scheimpflug photography. Acta Opbthalmol Scand. 2002;80:379-383.

37. Buehren T, Collins MJ, Carney L. Corneal aberrations and reading Optom Vis Sci. 2003;80(2):159-166.

38. Smolek MK. Interlamellar cohesive strength in the vertical meridian of human eye bank corneas. Invest Opbthalmol Vis Sci. 1993; 34(10):2962-2969.

39. David R, Zangwill L, Briscoe D, Dagan M, Yagev R, Yassur Y. Diurnal intraocular pressure variations: an analysis of 690 diurnal curves. Br J Ophthalmol. 1992;76:280-283.

40. Barbero $\mathrm{S}$. Refractive power of a multilayer rotationally symmetric model of the human cornea and tear film. J Opt Soc Am A. 2006;23(7):1578-1585. 